\title{
Relevância da temperatura e do esvaziamento gástrico de líquidos consumidos por praticantes de atividade física
}

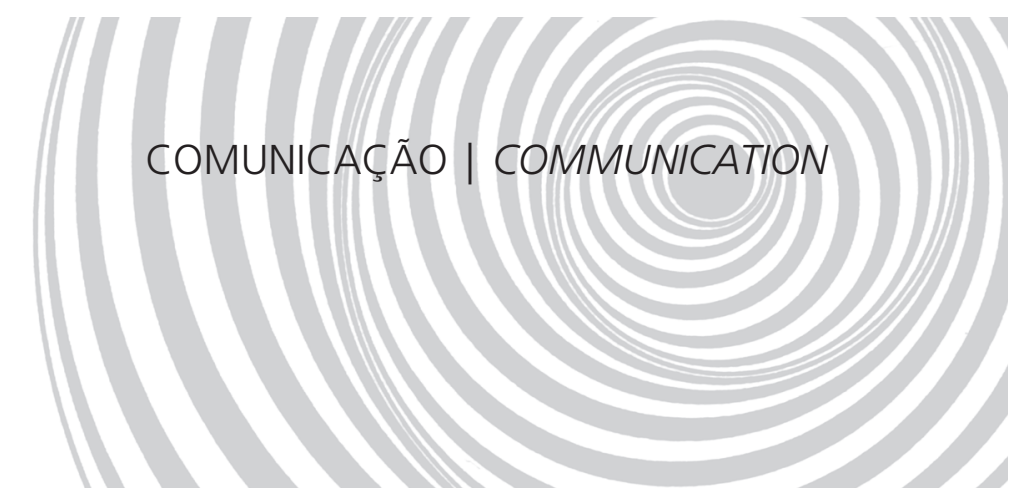

\author{
Relevance of temperature and gastric emptying \\ of liquids consumed by individuals who \\ practice physical activities
}

Rafael Pires da SILVA'

Janaína Lubiana ALTOÉ1

João Carlos Bouzas MARINS'

\section{RE S U M O}

A prática de atividade física implica alterações da homeostase hidroeletrolítica, que podem trazer impactos adversos ao desempenho do indivíduo e, talvez, à saúde, caso os líquidos corporais perdidos no suor são sejam suficientemente repostos. A eficiência da hidratação depende também do esvaziamento gástrico, que, por sua vez, é influenciado por vários fatores, dentre os quais a temperatura da solução ingerida. Este estudo objetiva analisar criticamente os procedimentos de reposição de líquidos adotados por praticantes de atividade física e discutir como o efeito da temperatura do líquido sobre o esvaziamento gástrico influencia esse comportamento de hidratação. Verificou-se que estudos relacionados ao tema apresentavam resultados conflituosos, que se devem, provavelmente, aos diferentes procedimentos metodológicos utilizados. Em condições de exercício, são insuficientes os trabalhos que avaliam a temperatura do líquido no trato gastrintestinal. Durante o repouso, observa-se que os efeitos de temperaturas extremas, considerando o tempo total de esvaziamento gástrico, não são significantes, uma vez que a temperatura intragástrica após a ingestão da bebida normaliza-se rapidamente. Contudo, existem evidências científicas claras de que o consumo de bebidas geladas aumenta o esvaziamento gástrico nos primeiros minutos após a ingestão. Este fato deve ser melhor estudado, quando associado a outros fatores pré-competição, como o estado psicológico do atleta. Entretanto, baixas temperaturas melhoram a palatabilidade da solução, implicando maior ação de hidratação pelos atletas, diminuindo o risco de desidratação. Os efeitos da baixa temperatura sobre o esvaziamento gástrico não são determinantes, tendo a reposição de líquidos fatores de intervenção mais relevantes do que a temperatura.

Termos de indexação: Esvaziamento gástrico. Exercício. Hidratação.

\footnotetext{
1 Universidade Federal de Viçosa, Departamento de Educação Física, Laboratório de Performance Humana. Av. P.H. Rolfs, s/n., 36571-000, Viçosa, MG, Brasil. Correspondência para/Correspondence to: R.P. SILVA. E-mail: <rafael.pires@ufv.br>.
} 


\section{A B S T R A C T}

Physical activity implies in changes to the water and electrolyte homeostasis which may result in adverse effects on performance and possibly on health if the body liquids lost by sweating are not completely replaced. Hydration efficiency also depends on gastric emptying, which is affected by several factors, including the temperature of the ingested liquid. The objective of this study was to critically assess the fluid replacement practices used by individuals who practice physical activities and discuss how the effect of liquid temperature on gastric emptying influences this hydrating behavior. Studies on the theme have found contradicting results, probably due to the different methods used. The studies that investigate liquid temperature in the gastrointestinal tract during exercise are limited. Considering the total gastric emptying time during rest, extreme liquid temperatures have no significant effect on the total gastric emptying time, since the temperature inside the stomach normalizes quickly after ingestion of the liquid. However, there are clear scientific evidences that the ingestion of ice-cold liquids increases gastric emptying in the first minutes after ingestion. This fact needs to be better investigated when it is associated with other pre-competition factors, such as the psychological state of the athlete. However, low temperatures improve the palatability of the drink, implying in greater consumption by the athletes and reducing the risk of dehydration. The effects of low temperature on gastric emptying are not decisive, since fluid replacement has intervention factors that are more relevant than temperature.

Indexing terms: Gastric emptying. Exercise. Fluid therapy.

\section{N T R O D U ÇÃ O}

A prática de atividade física expõe o indivíduo a uma elevação da temperatura corporal, que é mediada, entre outros fatores, pelo gasto energético, pelas condições ambientais e pelo tipo de vestimenta utilizada ${ }^{1}$. A liberação desse calor produzido se dá, primeiramente, através da evaporação do suor sobre a pele, o que atribui à sudorese um papel fundamental na manutenção do equilíbrio homeostático durante o exercício².

O suor contém água e eletrólitos que, se não forem apropriadamente repostos, podem favorecer o desenvolvimento de quadros de desidratação e hiponatremia, refletindo em prejuízos ao rendimento e até mesmo à saúde ${ }^{3}$. A desidratação, porém, é o distúrbio eletrolítico mais comum, podendo ser observado a partir de uma redução de, aproximadamente, $2 \%$ do peso corporal, resultando em aumento da temperatura interna, da freqüência cardíaca e da percepção de esforço, principalmente se a atividade se realizar sob forte calor $^{2,4}$. Uma hidratação adequada com água e eletrólitos antes, durante e após o treinamento e a competição torna-se, portanto, fundamental ao desempenho ${ }^{5}$.

Em atividades com características predominantemente aeróbicas, sobretudo aquelas com duração superior a uma hora, a ingestão de pequenas quantidades de carboidrato durante o exercício é também importante para manutenção da glicemia e dos estoques de glicogênio muscular em níveis necessários ao retardo da fadiga e à melhora do desempenho, isto é, favorecendo o aumento da intensidade ou a extensão do esforço ${ }^{6-8}$.

Uma hidratação eficiente garante condição ideal para que o atleta mantenha sua capacidade física, equilibrando a homeostase hidroeletrolítica, além de fornecer energia, sendo isso fundamental quanto maior for o tempo de exercício. Contudo, para que o carboidrato ingerido esteja disponível como fonte de energia, o líquido precisa, primeiramente, passar pelo estômago e ser absorvido no intestino, ou seja, o efetivo aproveitamento do líquido consumido vai depender da velocidade do efluxo gástrico ${ }^{9}$. Dessa forma, o esvaziamento gástrico é considerado um dos mais importantes fatores para determinar a disponibilidade e, portanto, a eficácia de uma bebida ingerida ${ }^{10,11}$.

O esvaziamento gástrico é influenciado durante o exercício por vários fatores, dos quais os mais importantes são: a) conteúdo energético da solução ${ }^{12}$; b) osmolaridade ${ }^{13,14}$; c) volume de líquido ingerido ${ }^{15,16}$; d) intensidade e tipo do exercício $^{17-19}$; sendo controversa a influência do tipo de 
exercício ${ }^{20}$; e) pH da solução21; f) nível de desidratação' . g) temperatura do líquido9,21. Entretanto, a influência da temperatura do líquido, como elemento potencializador ou inibidor do esvaziamento gástrico, vem sendo questionada ${ }^{18}$.

Os resultados são ainda mais limitados, quando se considera a influência da temperatura do líquido sobre o esvaziamento gástrico em condições de exercício. As posições antagônicas acerca da influência da temperatura baixa do líquido consumido sobre o esvaziamento gástrico geram questionamentos sobre a forma correta de orientar os praticantes de exercícios físicos sobre como proceder durante o planejamento de hidratação.

Frente às muitas informações e às dúvidas existentes sobre o tema, o objetivo deste estudo de revisão foi analisar os procedimentos de reposição de líquidos adotados por praticantes de atividade física e discutir como o efeito da temperatura do líquido sobre o esvaziamento gástrico influencia esse comportamento de hidratação.

\section{Panorama atual e implicações metodológicas}

Para alcançar os objetivos deste estudo foi redigida revisão apoiada em literatura científica publicada no período de 1968 a 2007, na base de dados Medline, PubMed, utilizando-se os termos gastric emptying, gastric temperature, fluid replacement, exercise, hydration, dehydration, assim como na base de dados SciELO, com os descritores esvaziamento gástrico, temperatura gástrica, exercício, hidratação e desidratação.

Diferentes métodos são empregados para o estudo do esvaziamento gástrico, os quais apresentam em sua extensão, vantagens e limitações. Dentre as técnicas não-invasivas, destacam-se a ultra-sonografia e a cintilografia, sendo esta última considerada o padrão ouro em análises clínicas desse tipo ${ }^{18,22}$. No entanto, o posicionamento estável que se deve assumir em testes dessa natureza torna sua aplicação durante o exercício inviável, além do risco de expor o sujeito à radiação ${ }^{13}$.

Os métodos utilizados durante o exercício são mais invasivos e envolvem o processo de intubação gástrica. Primeiramente, esta técnica fornece apenas o valor do volume gástrico residual obtido após um determinado tempo ${ }^{23}$. Isso gera um problema, pois, para que se tenha uma curva da resposta do indivíduo, é necessária a realização de vários testes no sujeito. Em razão de um mesmo indivíduo apresentar variações no esvaziamento gástrico em dias diferentes, foi desenvolvida uma técnica que permite múltiplas aferições durante o mesmo teste, proporcionando resultados mais completos $^{24}$. A double sampling intubation technique é um procedimento mais fidedigno, com boa reprodutibilidade ${ }^{25}$, e também o mais utilizado atualmente em condições de exercício; contudo, nem todas as pessoas se adaptam, além do fato de possuir todas as outras limitações de um teste invasivo.

$\mathrm{O}\left[{ }^{13} \mathrm{C}\right]$ - acetate breath test envolve a utilização de isótopos estáveis. É outra técnica não-invasiva e tem se mostrado promissora e eficaz, quando usada em situações de exercício. Van Nieuwenhoven et al. ${ }^{26}$, que validaram a técnica, encontraram correlação significante entre os valores Time-to-peak ${ }^{13} \mathrm{CO}_{2}$ enrichment de esvaziamento gástrico com os resultados Gastric emptying half-time apresentados pela técnica de intubação. No entanto, o teste apresentou a desvantagem de ser influenciado pelo tempo de absorção, pela taxa de oxidação e exalação do ${ }^{13} \mathrm{CO}_{2}$, ou seja, é possível, com a aplicação da técnica, afirmar se o esvaziamento gástrico reduz em determinada condição, mas é ineficaz para quantificar precisamente essa redução em termos de volume ${ }^{18}$.

As investigações envolvendo a temperatura do líquido e o esvaziamento gástrico perderam evidência no cenário científico a partir da segunda metade dos anos 90, o que impede a formação de um consenso sobre o real grau de influência da temperatura da bebida sobre a quantidade de fluido que é liberado para o intestino para ser absorvido 27,28 . 
Estudos que serão analisados a seguir encontraram resultados importantes no que se refere ao esvaziamento gástrico e à temperatura das bebidas. Tais resultados demasiadamente divergentes permitiram a formação de duas linhas distintas de pensamento: uma dos que acreditam haver interferência significativa da temperatura do líquido sobre o trato gastrintestinal e outra que apóia a irrelevância desses efeitos. Essas informações inconclusas e contraditórias se devem, provavelmente, aos diferentes procedimentos metodológicos utilizados, que, obviamente, dependendo da subjetividade ou do quão invasivos forem, vão apresentar resultados diferenciados. Outra possibilidade é quanto às diferenças na temperatura e no volume do líquido escolhido em cada estudo, tendo em vista que o volume da bebida influencia significativamente o esvaziamento gástrico ${ }^{15}$, o que pode mascarar os possíveis efeitos da temperatura. Da mesma forma, a escolha de diferentes faixas de temperaturas, que inclui valores extremos, pode também ter gerado resultados adversos.

\section{Influência da temperatura do líquido no esvaziamento gástrico}

\section{Estudos favoráveis}

Um estudo de referência clássica, que relata uma possível ação da temperatura sobre o trato gastrintestinal durante o exercício, foi realizado por Costill \& Saltin²1, no qual se postulou que soluções mais frias, com temperatura em torno de $5^{\circ} \mathrm{C}$, se esvaziavam mais rapidamente do que uma solução à temperatura de $35^{\circ} \mathrm{C}$, ou seja, o esvaziamento gástrico diminuiria à medida que a temperatura do líquido se elevasse ${ }^{9}$. Seguindo esse mesmo conceito, publicações posteriores apenas reproduziam essa concepção, sem se apoiar em estudos mais aprofundados e realmente comprovadores. Além disso, grande parte dos trabalhos que enfocaram essa temática foi realizada em condições de repouso. A partir das afirmações de Costill \& Saltin ${ }^{21}$, dificilmente se encontra ao alcance do cenário científico internacional o registro de outras investigações feitas em condições de exercício que abordem e confirmem essa tendência.

Estudos conduzidos com os avaliados em estado de repouso não só contradizem essa idéia, como ampliam o universo de discussão. À frente de um desses achados, Sun et al. ${ }^{29}$ concluíram que a bebida fria retardava significativamente 0 fluxo gástrico. As temperaturas das soluções utilizadas no estudo variavam de $4^{\circ} \mathrm{C}$ a $50^{\circ} \mathrm{C}$, com a bebida controle a $37^{\circ} \mathrm{C}$. Sessenta segundos após a ingestão da bebida a $50^{\circ}$ foi registrada a maior temperatura intragástrica $\left(43^{\circ} \mathrm{C}\right)$, enquanto a menor $\left(21,2^{\circ} \mathrm{C}\right)$ ocorreu 45 segundos após a ingestão da bebida mais fria.

A temperatura intragástrica retornou à temperatura corporal 20-30 minutos após a ingestão de ambos os líquidos, o que indica efeito da ação térmica no esvaziamento gástrico somente em um primeiro momento até esse tempo. Tanto a bebida quente como a fria demoraram mais tempo a passar pelo estômago dos avaliados do que a bebida controle $\left(37^{\circ} \mathrm{C}\right)$, porém somente foi verificada diferença significativa na inibição quando os valores da temperatura intragástrica foram menores, pela ação do líquido mais frio.

Outro estudo, também investigando indivíduos em estado de repouso mostrou significante retenção gástrica para o líquido de temperatura mais baixa $\left(4^{\circ} \mathrm{C}\right)$, que, possivelmente, se relacionava à redução dos peristaltismos gástricos ${ }^{30}$. Macedo et al. ${ }^{31}$ também verificaram que a administração de água gelada em sujeitos em repouso inibia o efluxo rápido de água do estômago, porém não observaram efeitos inibitórios cumulativos, quando ofereceram uma quantidade de água, seguida cinco minutos depois por outra, com temperatura mais baixa ou mais elevada que a primeira.

Foi possível verificar, em mais dois outros trabalhos ${ }^{32,33}$, ao serem utilizados procedimentos metodológicos semelhantes, que o corpo em repouso, quando submetido a estresse térmico de baixa temperatura, tem o esvaziamento gástrico 
do líquido significativamente retardado. Esse estresse era estabelecido com sucessivas imersões contínuas de um minuto da mão não-dominante dos voluntários em água a $4^{\circ} \mathrm{C}$, com intervalos de 15 segundos a cada imersão, em um tempo total de 20 minutos. A aplicação do procedimento anterior teria levado a uma série de modificações na organização do sistema nervoso entérico, influenciando principalmente as alterações na propagação das ondas de pressão do antro, do piloro e do duodeno ${ }^{34}$. Essas mudanças na mobilidade duodenal e pilórica, portanto, favoreceram o retardo do esvaziamento gástrico quando o corpo teve contato com temperaturas baixas.

Ao analisarem o esvaziamento gástrico em repouso após o consumo de $500 \mathrm{~mL}$ de uma bebida carboidratada a $5 \%$ em quatro temperaturas diferentes $\left(4^{\circ} \mathrm{C}, 20^{\circ} \mathrm{C}, 37^{\circ} \mathrm{C}\right.$ e $\left.55^{\circ} \mathrm{C}\right)$, Troncon \& lazigi ${ }^{35}$ verificaram que a temperatura elevada da bebida também poderia interferir no sistema gástrico. Em comparação ao líquido a $37^{\circ} \mathrm{C}$, o esvaziamento gástrico foi lento, quando se consumiu o líquido a $4^{\circ} \mathrm{C}$ quanto a $55^{\circ} \mathrm{C}$, porém foi estatisticamente significante somente para o último.

Os resultados, portanto, claramente divergentes, mostram que tanto bebidas mais quentes ou mais frias do que a temperatura corporal afetariam o esvaziamento gástrico, inibindo-o. Essa inibição se daria, principalmente, por alterações na organização da pressão das ondas antropilóricas e do estímulo das ondas de pressão isoladas do piloro ${ }^{36}$. Sabe-se que o sistema nervoso entérico, por meio de sua complexa rede de receptores e hormônios, tem importante papel na regulação do esvaziamento gástrico ${ }^{37}$, no entanto, não há como afirmar, seguramente, o processo pelo qual a temperatura alta ou baixa do líquido afetaria essa atividade nervosa.

Pode-se concluir, ainda, que seja simplesmente problemática qualquer tentativa de reprodutibilidade desses resultados ou procedimentos metodológicos na condição de exercício. Mesmo tendo em vista as indicações de que não existe diferença significativa entre o esvaziamento gástrico em repouso frente a uma condição de exercício a uma intensidade de até $75 \%$ do consumo máximo de oxigênio $\left(\mathrm{VO}_{2} \mathrm{max}\right)^{18}$, as condições fisiológicas durante o exercício implicam aumento da temperatura central, incluindo a temperatura de todo o trato gástrico. Somente esse fator já é suficiente para promover redução da diferença de temperatura entre o líquido e as paredes estomacais. Desde o primeiro momento de contato com a boca até a chegada no estômago, o líquido fará uma troca constante de temperatura, sendo extremamente improvável que atinja o centro do estômago à mesma temperatura da ingestão, vindo ainda influir, acelerando ou atrasando o esvaziamento gástrico.

Outro elemento que torna difícil uma comparação entre os estudos de esvaziamento gástrico em condições de repouso e em exercício é o fator comportamental. Dificilmente se adequaria à realidade a existência de um atleta fazendo uso de uma bebida a $55^{\circ} \mathrm{C}$ e competindo em condições de extremo calor. Da mesma forma, haveria dificuldades em fazer com que um atleta que se exercitasse sob as baixas temperaturas dos invernos rigorosos, como os da Europa, se habituasse a ingerir líquidos a $4^{\circ} \mathrm{C}$.

Mesmo diante de condições ambientais extremas, combinadas à utilização dessas altas margens de temperatura, fosse registrado significante aumento do efluxo gástrico, que reproduzisse uma grande vantagem para o atleta, é necessário enfatizar que o esvaziamento gástrico é somente a primeira etapa do processo de hidratação. Fica a cargo do intestino a absorção de todo o fluido que deixa o estômago e passa ao duodeno. O líquido precisa, também, ser absorvido na luz intestinal para uma hidratação eficaz, isto é, para que água e minerais sejam repostos e que o carboidrato esteja disponível na corrente sangüínea para oxidação. Portanto, não se traduz em vantagem aumentar em demasia o efluxo gástrico, se a absorção intestinal não se revela da mesma forma eficiente. 


\section{Estudos contrários}

Alguns pesquisadores não atribuem um papel importante à temperatura do líquido no esvaziamento gástrico, ou os seus efeitos relacionados seriam minimamente influenciadores ${ }^{18,28}$. McArthur \& Feldman ${ }^{38}$ não encontraram, em repouso, diferença na taxa de esvaziamento gástrico de uma solução em três temperaturas distintas $\left(58^{\circ} \mathrm{C}, 37^{\circ} \mathrm{C}\right.$, e $4^{\circ} \mathrm{C}$ ), verificando o retorno da temperatura intragástrica aos valores normais 16,7 e 23,8 min após a ingestão da bebida mais quente e da mais fria, respectivamente.

Bateman ${ }^{39}$ analisou o comportamento gástrico de oito sujeitos em repouso, durante 60 minutos após ingestão de 200 ou $500 \mathrm{~mL}$ de líquido, ora a $12^{\circ} \mathrm{C}$, ora a $37^{\circ} \mathrm{C}$, e, também constatou que o esvaziamento gástrico foi ligeiramente maior somente nos primeiros minutos depois da ingestão da bebida mais fria, não havendo interferências significativas posteriores. Os resultados deste estudo revelam, ainda, dados extremos e altos valores de desvio-padrão, o que chama a atenção para uma considerável relevância das características individuais dos sujeitos, no que se refere à qualidade do esvaziamento gástrico para líquidos em diferentes temperaturas.

Resultados semelhantes foram discutidos por Shi et al. ${ }^{28}$, ao analisarem os efeitos do consumo de $481 \mathrm{ml}$ de bebida carboidratada contendo frutose, glicose, sacarose ou sacarose + frutose, em temperatura de $12^{\circ} \mathrm{C}$, sobre o esvaziamento gástrico durante o repouso. Um minuto depois da ingestão da bebida, a temperatura intragástrica caiu consideravelmente e retornou para $30^{\circ} \mathrm{C}$ cinco minutos após. Depois de 20 minutos, os níveis térmicos corporais já estavam praticamente próximos da normalidade. Esses resultados podem ser mais bem observados na Figura 1.

Os resultados mostraram que a taxa de esvaziamento gástrico foi um pouco mais elevada nos primeiros 10 minutos do que durante os $20 \mathrm{e}$ 30 minutos seguintes, quando a temperatura intragástrica estava quase próxima do normal. Além disso, o declínio posterior da taxa de

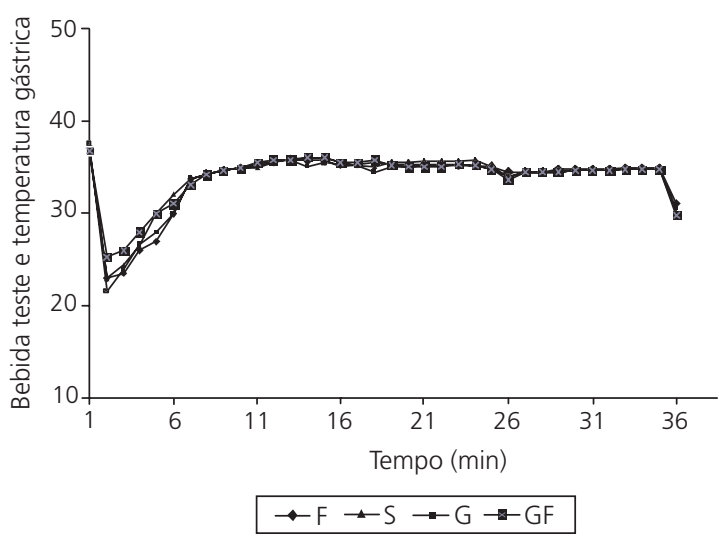

Figura 1. Variações na temperatura gástrica de indivíduos em repouso $(n=8)$ após a ingestão de soluções contendo frutose $(F)$, glicose $(G)$, sacarose $(S)$ ou glicose e frutose (GF).

Fonte: Adaptado de Shi et al. ${ }^{28}$.

esvaziamento gástrico, aparentemente, se relacionou mais com a queda do volume gástrico do que com a temperatura, ou seja, o volume gástrico seria mais importante do que a temperatura da bebida para efeito regulador do esvaziamento gástrico ${ }^{28}$.

A partir dos dados anteriormente apresentados, pode-se dizer, então, que o líquido em temperatura mais baixa teria um mínimo efeito sobre o esvaziamento gástrico. Essa ação se daria somente nos primeiros minutos da ingestão, enquanto a temperatura intragástrica é significativamente baixa para proporcionar alguma alteração ${ }^{36}$. No entanto, outros fatores intervenientes no controle gástrico podem inibir uma possível ação da temperatura do líquido, como é o caso do volume.

Em situações pré-exercício, como nos minutos que antecedem uma largada na maratona, ou a concentração no vestiário antes de uma partida de futebol, o atleta, na maioria das vezes, se apresenta nervoso, com níveis elevados de tensão e ansiedade, o que tem ação direta sobre o esvaziamento gástrico, retardando-o ${ }^{18}$. Seria, portanto, interessante que a bebida utilizada nesses momentos, que tem como objetivo a manutenção dos níveis glicêmicos e a preservação do glicogênio muscular, não fosse administrada em temperaturas 
extremas, para que o esvaziamento gástrico não sofresse simultaneamente, ação de dois agentes intervenientes: o estresse do atleta pré-competição e a temperatura do líquido. Mesmo que os efeitos da temperatura sejam mínimos e transitórios, quando associado a outro fator, como o estado emocional do atleta, podem ser prejudiciais. No entanto, é necessário ressaltar a grande participação das características individuais em todo esse processo, o que torna importante o treinamento desse tipo de ação antes do dia da competição, permitindo, assim, testar qual é a temperatura da bebida que irá realmente trazer vantagens ou desvantagens para determinado atleta.

Nota-se, entretanto, claramente a carência de confiabilidade nos estudos conduzidos durante o exercício e que têm como foco principal a análise da temperatura da solução de hidratação sobre o trato gastrintestinal. Brouns ${ }^{18}$, discorrendo sobre os diversos fatores que interferem no esvaziamento gástrico durante o exercício, posicionou-se contra a idéia de uma possível ação significante da temperatura sobre o esvaziamento gástrico. No mesmo estudo, os atletas são aconselhados a ajustarem a temperatura do líquido de acordo com a preferência e tolerância individual. Ressalta-se, também, que as bebidas geladas podem não trazer benefícios ao esvaziamento gástrico, mas colaborar na retirada do calor do corpo, e que, ao contrário, quando o exercício se der em temperaturas mais baixas, as bebidas com temperatura um pouco mais elevada sejam mais indicadas, por também oferecerem benefícios termorregulativos.

\section{Temperatura do líquido na relação com a palatabilidade}

A temperatura do líquido se relaciona fortemente às estratégias de hidratação, uma vez que também é fator responsável pelo aumento da palatabilidade' 1 . Em comparação com uma bebida em temperatura ambiente, a bebida mais fria, entre $6^{\circ}$ e $22^{\circ} \mathrm{C}$, se torna mais palatável em situação de exercício, sobretudo se a atividade estiver sendo realizada no calor ${ }^{40}$. Esse aumento da palatabilidade pela temperatura presume que a bebida mais refrigerada tende a ser consumida em maiores quantidades durante o exercício ${ }^{9,41}, 0$ que incide sobre uma também maior e melhor taxa de hidratação, resultando, assim, na diminuição dos efeitos nocivos da desidratação.

Promover maior consumo de líquidos por parte dos atletas se torna, de certa forma, interessante, uma vez que o controle dos líquidos corporais nem sempre é possível, já que os atletas, freqüentemente, se hidratam com quantidade muito abaixo da capacidade de esvaziamento gástrico, que pode chegar a 1 litro por hora ${ }^{42,43}$.

O nível de conhecimento e a caracterização das práticas de hidratação de atletas de diferentes modalidades tem sido investigado ${ }^{44-46}$. Dos 135 caratecas observados em estudo, apenas $53,13 \%$ utilizavam algum tipo de líquido na competição, e 47,06\% durante o período de treinamento, no qual a perda hídrica é mais considerável nessa modalidade ${ }^{45}$. Com base nas recomendações do Colégio Americano de Medicina do Esporte $^{42}$ (ACSM), Brito \& Marins ${ }^{46}$ identificaram que os procedimentos de hidratação adotados por judocas também não eram pertinentes, sendo, ainda, que a maior parte dos atletas, nunca teve nenhum tipo de orientação técnica sobre o assunto.

A situação de pouca reposição líquida também foi observada em $14,3 \%$ dos maratonistas brasileiros, que responderam não ter o costume de se hidratar ${ }^{47}$, e em atletas universitários das mais variadas modalidades (basquetebol, voleibol, futebol, handebol, natação, dança, ciclismo, entre outros) ${ }^{44}$, em que se destaca um percentual de $9 \%$ que nunca ou quase nunca se hidratam durante o treinamento, em competições esse valor passa para 5,1\%. Esse comportamento aumenta consideravelmente o risco de desidratação, o que, certamente, diminuirá a capacidade de treinamento ou de competição do atleta.

Os estudos acima citados relatam diversas razões que levam os atletas a se hidratarem em quantidades não adequadas, entre eles: descon- 
forto gástrico, palatabilidade, ausência de orientação por parte dos técnicos e preparadores físicos, o alto custo de alguns repositores hidroeletrolíticos, ou, simplesmente, por considerarem a hidratação irrelevante. Percebe-se, portanto, que apesar de a palatabilidade não ser o principal motivo pelo qual os atletas se hidratam pouco, uma bebida mais palatável poderá contribuir para aumentar o consumo de líquidos e diminuir as chances do aparecimento de um quadro de desidratação'.

Em outro estudo, atletas foram questionados sobre as estratégias de hidratação que usualmente adotam, e, mais de $70 \%$ dos triatletas, ciclistas e corredores de fundo entrevistados revelaram não se importar com a temperatura do líquido com o qual se hidratam, atentando apenas em não ingerir líquidos em temperaturas demasiadamente baixas, uma vez que, segundo os atletas, isso causa grande mal-estar ${ }^{48}$. A Tabela 1 apresenta a distribuição percentual de preferência da temperatura do líquido de atletas brasileiros de diferentes modalidades, em que se pode observar maior concentração das respostas para líquidos refrigerados.

Mundel et al. ${ }^{51}$ observaram que, durante o exercício contínuo em bicicleta a $34^{\circ} \mathrm{C}$, realizado até a exaustão, o líquido a $4^{\circ} \mathrm{C}$ foi significativamente mais consumido do que a uma temperatura mais neutra $\left(19^{\circ} \mathrm{C}\right)$, corroborando os resultados encontrados por Sandick et al. ${ }^{52}$, segundo os quais os indivíduos, após o exercício, também preferiram a ingestão de uma bebida que tivesse a temperatura mais baixa, sendo ingerido um maior volume de água a $5^{\circ} \mathrm{C}$, comparado às outras temperaturas $\left(16,22\right.$ e $\left.38^{\circ} \mathrm{C}\right)$.
Vale ressaltar, entretanto, que o atleta deve estar atento para que o aumento da palatabilidade de uma bebida não induza a um consumo exagerado de líquido. O over consumption, como é chamada essa ingestão irregular com tendência ao excesso, pode levar a desconfortos gastrintestinais e também à formação de quadros de hiponatremia, associados ao grande consumo de água, que provocará diluição do sódio plasmático. A fim de evitar problemas como esses, o ACSM ${ }^{1}$ recomenda aos atletas monitorarem a variação do peso corporal antes e após o treinamento e a competição, de modo que o volume de líquido perdido sirva como referencial para a quantidade que deve ser reposta.

Algumas proposições têm sido feitas em relação à temperatura da bebida na hidratação; no entanto, as referências se justificam mais pela palatabilidade do que por implicações no esvaziamento gástrico. A proposta de hidratação recomendada pelo $\mathrm{ACSM}^{42}$ preconiza que o volume de líquido ingerido em intervalos regulares ao longo do exercício, deve estar em uma temperatura palatável, entre 15 e $22^{\circ} \mathrm{C}$. A mais recente publicação do $\mathrm{ACSM}^{1}$ sobre a temática da reposição hídrica reafirma sua posição quanto à faixa de temperatura e seus benefícios à palatabilidade, acrescentando ainda que, tanto a temperatura como a presença de sabor nas soluções, são preferências que variam grandemente de acordo com os indivíduos e as culturas' ${ }^{1}$. A diretriz da Sociedade Brasileira de Medicina do Esporte ${ }^{5}$ e o consenso estabelecido pelo Gatorate Sports Science Institute (GSSI) na América Latina ${ }^{53}$ concordam com as estratégias de hidratação estabelecidas pelo $\mathrm{ACSM}^{42}$ e salientam ainda que, além de uma

Tabela 1. Relação de estudos sobre o percentual da preferência individual da temperatura do líquido entre atletas.

\begin{tabular}{lccccc}
\hline & Marins \& Ferreira ${ }^{44}$ & Brito et al. ${ }^{45}$ & Brito \& Marins $^{46}$ & Ferreira $^{49}$ & Brito et al. $^{50}$ \\
\hline & Atletas universitários & Caratecas & Judocas & Corredores de rua & Praticantes de Jiu-Jítsu \\
Extremamente gelado & 6,77 & 6,67 & 3,23 & 1,04 & 9 \\
Moderadamente gelado & 63,54 & 53,33 & 63,59 & 47,15 & 56 \\
Ambiente & 39,09 & 37,04 & 33,18 & 51,81 & 35 \\
\hline
\end{tabular}

*Atletas universitários (basquetebol, pólo-aquático, voleibol, futsal, handebol, futebol, judô, jiu-jítsu, ciclismo, natação e dança). 
temperatura entre 15 e $22^{\circ} \mathrm{C}$, a solução deve apresentar sabor que seja adequado às preferências individuais do sujeito, a fim de que as suas necessidades de líquido sejam atingidas mais facilmente.

Com base em trabalhos anteriores, é possível recomendar que a temperatura do líquido consumido nas situações de exercício, tanto água como solução carboidratada, seja indicada tomando como base as condições ambientais nas quais o exercício é realizado, a fim de aproveitar ao máximo dos benefícios termorregulativos proporcionados. Ou seja, durante provas realizadas em climas frios, a administração de uma solução com uma temperatura palatável, em torno dos $19^{\circ} \mathrm{C}$, seria interessante, enquanto uma temperatura mais baixa $\left(12^{\circ} \mathrm{C}\right)$ seria bem mais indicada para as situações de competições e treinos realizados sob calor intenso. Em condições ambientais termoneutras, pode-se optar pelo uso de uma temperatura em que prevaleça a preferência individual do atleta.

\section{O N C L U S Ã O}

Os efeitos da temperatura em situações de repouso, considerando o tempo total de esvaziamento gástrico, são considerados mínimos, uma vez que a temperatura intragástrica após a ingestão da bebida normaliza-se rapidamente, inibindo qualquer ação mais intensa da temperatura do líquido.

Soluções geladas ingeridas em repouso aumentam ligeiramente o esvaziamento gástrico, e, mesmo que seus efeitos não sejam significativos, deve-se evitar o uso de soluções com temperaturas extremas em situações pré-exercício, a fim de não colaborar com os efeitos nocivos do estado emocional do atleta no trato gastrintestinal. Contudo, bebidas refrigeradas promovem o aumento da palatabilidade; logo, o seu consumo deve ser monitorado, procurando sempre manter um equilíbrio hídrico.

Recomenda-se que a temperatura do líquido seja indicada tomando como base a condição do ambiente, oferecendo-se bebidas mais geladas em situações de clima quente, não tão geladas em clima frio e mantendo ainda a preferência individual do atleta em ambientes temperados.

Frente ao desenvolvimento de novas tecnologias invasivas, sugere-se, ainda, a necessidade de realização de mais estudos sobre a temperatura do líquido e o esvaziamento gástrico, considerando as mais variadas formas de exercício, devido à escassez de dados atualizados e à impossibilidade de reproduzir, em condições de exercício, os resultados obtidos durante o repouso.

\section{COLABORADORES}

R.P. SILVA contribuiu com a busca de referências bibliográficas, coleta e organização dos dados, a análise, a interpretação dos resultados e a discussão dos limites e alcances metodológicos relativos ao tema. J.L. ALTOÉ contribuiu com a busca de referências bibliográficas, coleta e organização dos dados, análise e a discussão dos resultados e a formatação do texto. J.C.B. MARINS orientador do trabalho atuou na coordenação, na análise e interpretação dos dados e na revisão do manuscrito.

\section{REFERÊ NCIAS}

1. American College of Sports Medicine. Position stand: exercise and fluid replacement. Med Sci Sports Exerc. 2007; 39(2):377-90.

2. Marins JCB, Dantas E, Navarro S. Deshidratación y ejercicio físico. Selección. 2000; 9(3):149-63.

3. Casa DJ, Clarkson PM, Roberts WO. American College of Sports Medicine roundtable on hydration and physical activity: consensus statements. Curr Sports Med Rep. 2005; 4(3): 115-27.

4. Cheuvront S, Carter R, Sawka MN. Fluid balance and endurance exercise performance. Curr Sports Med Rep. 2003; 2(4):202-8.

5. Sociedade Brasileira de Medicina do Esporte. Diretriz da Sociedade Brasileira de Medicina do Esporte. Modificações dietéticas, reposição hídrica, suplementos alimentares e drogas: comprovação de ação ergogênica e potenciais riscos para a saúde. Rev Bras Med Esporte. 2003; 9(2):1-13. 
6. Byrne C, Lim CL, Chew SA, Ming ET. Water versus carbohydrate-electrolyte fluid replacement during loaded marching under heat stress. Mil Med. 2005; 170(8):715-21.

7. Nicholas CW, Tsintzas K, Boobis L, Williams C. Carbohydrate-electrolyte ingestion during intermittent high-intensity running. Med Sci Sports Exerc. 1999; 31(9):1280-6.

8. Lima-Silva AE, Fernandes TC, De-Oliveira FR, Nakamura FY, Gevaerd MS. Metabolismo do glicogênio muscular durante o exercício físico: mecanismos de regulação. Rev Nutr. 2007; 20(4):417-29. doi: 10.1590/S141552732007 000400009.

9. Puhl SM, Buskirk ER. Bebidas nutrientes para o exercício e o esporte. In: Wolinsky I, Hikson JFJ. Nutrição no exercício e no esporte. 2a. ed. São Paulo: Roca; 2002. p.331-76.

10. Murray R. The effects of consuming carbohydrate-electrolyte beverages on gastric emptying and fluid absorption during and following exercise. Sports Med. 1987; 4(5):322-51.

11. Rehrer NJ, Beckers E, Brouns F, Ten Hoor F, Saris WHM. Exercise and training effects on gastric emptying of carbohydrate beverages. Med Sci Sports Exerc. 1989; 21(5):540-9.

12. Calbet JA, MacLean DA. Role of caloric content on gastric emptying in humans. J Physiol. 1997; 498(Pt 2):553-9.

13. Takii $H$, Takii Nagao $Y$, Kometani $T$, Nishimura T, Nakae T, Kuriki T, et al. Fluids containing a highly branched cyclic dextrin influence the gastric emptying rate. Int J Sports Med. 2005; 26(4): 314-9.

14. Vist GE, Maughan RJ. The effect of osmolality and carbohydrate content on the rate of gastric emptying of liquids in man. J Physiol. 1995; 486 (Pt 2):523-31.

15. Noakes TD, Rehrer NJ, Maughan RJ. The importance of volume in regulating gastric emptying. Med Sci Sports Exerc. 1991; 23(3):307-13.

16. Mitchell JB, Voss KW. The influence of volume on gastric emptying and fluid balance during prolonged exercise. Med Sci Sports Exerc. 1991; 23(3):314-9.

17. Leiper JB, Nicholas CW, Ali A, Williams C, Maughan RJ. The effect of intermittent high-intensity running on gastric emptying of fluids in man. Med Sci Sports Exerc. 2005; 37(2):240-7.

18. Brouns F. Gastric emptying as a regulatory factor in fluid uptake. Int J Sports Med. 1998; 19(Suppl 2):125-8.

19. Rehrer NJ, Brouns F, Beckers EJ, Ten Hoor F, Saris WH. Gastric emptying with repeated drinking during running and bicycling. Int J Sports Med. 1990; 11(3):238-43.

20. Houmard JA, Egan PC, Johns RA, Neufer PD, Chenier TC, Israel RG. Gastric emptying during $1 \mathrm{~h}$ of cycling and running at $75 \% \mathrm{VO}^{2}$ max. Med Sci Sports Exerc. 1991; 23(3):320-5.

21. Costill DL, Saltin B. Factors limiting gastric emptying during rest and exercise. J Appl Physiol. 1974; 37(5):679-83.

22. Valadares $C P$, Silva RAP, Tavares-Junior WC, Duarte MA. Apresentação da técnica de estudo do tempo de esvaziamento gástrico por meio da ultra-sonografia. Radiol Bras. 2006; 39(1):15-18.

23. George JD. New clinical method for measuring the rate of gastric emptying: the double sampling test meal. Gut. 1968; 9(2):237-42.

24. Beckers EJ, Rehrer NJ, Brouns F, Ten Hoor F, Saris $\mathrm{WH}$. Determination of total gastric volume, gastric secretion and residual meal using the double sampling technique of George. Gut. 1988; 29(12): 1725-29.

25. Beckers EJ, Rehrer NJ, Saris WH, Brouns F, Ten Hoor $F$, Kester AD. Daily variation in gastric emptying when using the double sampling technique. Med Sci Sports Exerc. 1991; 23(10):1210-2.

26. van Nieuwenhoven MA, Wagenmakers AJ, Senden JM, Brouns F, Brummer RJ. Performance of the [13C]-acetate gastric emptying breath test during physical exercise. Eur J Clin Invest. 1999; 29(11): 922-8.

27. Lambert GP, Bleiler TL, Chang RT, Johnson AK, Gisolfi CV. Effects of carbonated and noncarbonated beverages at specific intervals during treadmill running in the heat. Int J Sport Nutr. 1993; 3(2):177-93.

28. Shi X, Bartoli W, Horn M, Murray R. Gastric emptying of cold beverages in humans: effect of transportable carbohydrates. Int J Sport Nutr Exerc Metab. 2000; 10(4):394-403.

29. Sun WM, Houghton LA, Read NW, Grundy DG, Johnson AG. Effect of meal temperature on gastric emptying of liquids in man. Gut. 1988; 29(3): 302-5.

30. Collares EF, Brasil MR. Gastric emptying in children. I. Influence of the temperature of a hydration solution for oral use. Arq Gastroenterol. 1981; 18(3):123-6.

31. Macedo Ada S, Collares EF, Troncon LE, Machado NC. Gastric emptying in children. VI. Evaluation of the cumulative effect of the water low temperature. Arq Gastroenterol. 1986; 23(3):184-8.

32. Thompson DG, Richelson E, Malagelada JR. Perturbation of upper gastrointestinal function by cold stress. Gut. 1983; 24(4):277-83. 
33. Nakae $Y$, Kagaya M, Takagi R, Matsutani Y, Horibe $\mathrm{H}$, Kondo T. Cold pain prolongs gastric emptying of liquid but not solid meal: an electrical impedance tomography (EIT) study. J Gastroenterol. 2000; 35(8):593-7.

34. Fone DR, Horowitz M, Maddox A, Akkermans LM, Read NW, Dent J. Gastroduodenal motility during the delayed gastric emptying induced by cold stress. Gastroenterology. 1990; 98(5 Pt 1):1155-61.

35. Troncon LE, lazigi N. Effect of test meal temperature on the gastric emptying of liquids. Braz J Med Biol Res. 1988; 21(1):57-60.

36. Sun WM, Penagini R, Hebbard G, Malbert C, Jones $\mathrm{KL}$, Emery $\mathrm{S}$, et al. Effect of drink temperature on antropyloroduodenal motility and gastric electrical activity in humans. Gut. 1995; 37(3):329-34.

37. Thompson DG, Richelson E, Malagelada JR. Perturbation of gastric emptying and duodenal motility through the central nervous system. Gastroenterology. 1982; 83(6):1200-6.

38. McArthur KE, Feldman M. Gastric acid secretion, gastrin release, and gastric emptying in humans as affected by liquid meal temperature. Am J Clin Nutr. 1989; 49(1):51-4.

39. Bateman DN. Effects of meal temperature and volume on the emptying of liquid from the human stomach. J Physiol. 1982; 331(10):461-7.

40. Armstrong LE, Hubbard RW, Szlyk PC, Matthew WT, Sils IV. Voluntary dehydration and electrolyte losses during prolonged exercise in the heat. Aviat Space Environ Med. 1985; 56(8):765-70.

41. Hubbard RW, Sandick BL, Matthew WT, Francesconi RP, Sampson JB, Durkot MJ, et al. Voluntary dehydration and alliesthesia for water. J Appl Physiol. 1984; 57(3):868-73.

42. American College of Sports Medicine. Position stand: exercise and fluid replacement. Med Sci Sports Exerc. 1996; 28(1):i-vii.

43. Burke LM. Nutritional needs for exercise in the heat. Comp Biochem Physiol A Mol Integr Physiol. 2001; 128(4):735-48.
44. Marins JCB, Ferreira FG. Nível de conhecimento dos atletas universitários da UFV sobre hidratação. Fit Perform J. 2005; 4(3):175-87.

45. Brito ISS, Brito CJ, Fabrini SP, Marins JCB. Caracterização das práticas de hidratação em Karatecas do estado de Minas Gerais. Fit Perform J. 2006; 5(1):24-30.

46. Brito CJ, Marins JCB. Caracterização das práticas sobre hidratação em atletas da modalidade de judô no estado de Minas Gerais. Rev Bras Ciên Mov. 2005; 13(2):59-74.

47. Duarte MFS, Duarte CR, Andrade DR. Perfil de ultramaratonista brasileiros. Treinamento Desport. 1997; 3(2):65-8.

48. Marins JCB, Argudo C, Iglesias ML, Marins N, Zamora S. Hábitos de hidratación en un colectivo de pruebas de resistência. Selecciõn. 2004; 13(1): 18-28.

49. Ferreira FG. Hidratação e perda hidromineral em corredores e indivíduos ativos [dissertação]. Viçosa: Universidade Federal de Viçosa; 2007.

50. Brito ISS, Dinis A, Brito CJ, Marins, JCB. Caracterização das práticas e hábitos de hidratação em lutadores brasileiros de jiu-jitsu. In: Fontoura $P_{1}$ organizador. Coleção pesquisa em educação física. Jundiaí: Fontoura; 2007. p.153-60.

51. Mundel T, King J, Collacott E, Jones DA. Drink temperature influences fluid intake and endurance capacity in men during exercise in a hot, dry environment. Exp Physiol. 2006; 91(5):925-33.

52. Sandick BL, Engell DB, Maller O. Perception of drinking water temperature and effects for humans after exercise. Physiol Behav. 1984; 32(5):851-5.

53. Gatorade Sports Science Institute (GSSI). Consenso do Conselho Consultivo em Ciência e Educação do GSSI na América Latina. Atividade física no calor: regulação térmica e hidratação, 1999 [acesso 2007 ago 30]. Disponível em: <http://www.gssi.com.br>.

Recebido em: 9/10/2007

Versão final reapresentada em: 13/8/2008

Aprovado em: 22/4/2009 